\title{
Papers
}

\section{Within pair association between birth weight and blood pressure at age 8 in twins from a cohort study}

\author{
Terence Dwyer, Leigh Blizzard, Ruth Morley, Anne-Louise Ponsonby
}

\begin{abstract}
Objectives To study the association between birth weight and blood pressure in children from multiple pregnancies (multiplets), mostly twins, to determine whether maternal or genetic factors are responsible for the association.

Design Cohort study.

Setting Southern Tasmania.

Subjects 888 children including 104 multiplets (32 monozygotic, 72 dizygotic).

Main outcome measure Systolic blood pressure ( $\mathrm{mm}$ $\mathrm{Hg}$ ).

Results Blood pressure decreased with birth weight and increased with current body mass. After adjustment for age and body mass, systolic blood pressure changed by $-1.94 \mathrm{~mm} \mathrm{Hg}(95 \%$ confidence interval -2.89 to -0.98 ) per $1 \mathrm{~kg}$ increase in birth weight of singletons. For multiplets, blood pressure changed by $-7.0 \mathrm{~mm} \mathrm{Hg}(-10.1$ to -3.9$)$ for each $1 \mathrm{~kg}$ increase in birth weight. This was little altered in within pair analyses $(-5.3,-13.8$ to 3.2$)$ and was similar for both monozygotic $(-6.5,-22.5$ to 9.4$)$ and dizygotic $(-4.9,-15.8$ to 6.0$)$ pairs.

Conclusion Because the association between birth weight and blood pressure was largely unchanged in within pair analyses, exposures originating in the mother (such as nutritional status) cannot be wholly responsible. The association also remained within monozygotic pairs, suggesting that genetic predisposition is not wholly responsible either. The principal causal pathway must concern mechanisms within the fetoplacental unit. The stronger association in multiplets suggests that factors adversely influencing both blood pressure and birth weight are more prevalent in multiple pregnancies.
\end{abstract}

\section{Introduction}

Much evidence suggests that low birth weight is associated with high blood pressure ${ }^{1}$ and high risk of both cardiovascular disease ${ }^{2}$ and non-insulin dependent diabetes mellitus (type 2 diabetes) in adult life. ${ }^{3}$ Furthermore, risk factors for cardiovascular disease, including high mean blood pressure and glucose intolerance, have been shown to be more prevalent in childhood among those who were of low birth weight. ${ }^{145}$ The general proposition underlying the "fetal origins" hypothesis ${ }^{6}$ is that low birth weight is principally due to a poor nutrient supply to the fetus, ${ }^{7}$ which in turn may result from suboptimal maternal nutrient intake. Undernutrition at a critical period in fetal life may "programme" a permanent change in the structure or function of an individual, ${ }^{78}$ altering the distribution of cell types or gene expression, or both. ${ }^{9}$ This hypothesis has been supported by studies of rodents. ${ }^{10}$

Alternatively some factors other than nutrition may determine birth weight and influence the causal pathway leading to high blood pressure or high risk of either cardiovascular disease or type 2 diabetes. Environmental causes, particularly those associated with socioeconomic status, must be considered. ${ }^{11} 12$ Factors originating in the fetus such as genetic predisposition to both low birth weight and hypertension, type 2 diabetes, or cardiovascular disease could also potentially explain the association. In humans, experimental studies to test the specific hypotheses generally are not feasible for ethical and practical reasons. Genetic and extrauterine environmental causes can, however, be separated through studies of monozygotic and dizygotic subjects from multiple pregnancies (multiplets). This approach has not been reported previously in studies of the association between birth weight and blood pressure.

We had the opportunity to examine the association between birth weight and childhood blood pressure in both singletons and multiplets (mostly twins) in the Tasmanian infant health survey cohort of infants. Among multiplets we were able to make comparisons within pairs, for which the infants are matched on exposures of maternal origin. The underlying hypotheses for the within pair analyses were that if maternal factors are responsible for programming high blood pressure, the association would be weakened by pairing, and, if genetic predisposition is responsible, the association would be substantially reduced in monozygotic pairs but less so in dizygotic pairs.

\section{Subjects and methods}

\section{Subjects}

The Tasmanian infant health survey, a cohort study initiated to study sudden infant death syndrome, provided standard information prospectively on around one in five births in Tasmania from 1988 to $1995 .{ }^{13}$ Selection of singleton births was on the basis of a scoring system using six risk factors for sudden infant

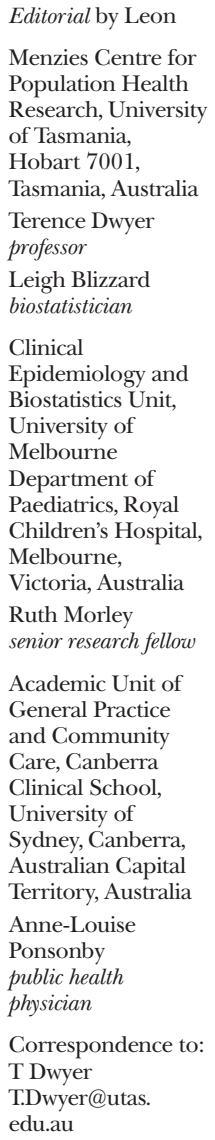

BMJ 1999;319:1325-9 
death syndrome (young maternal age, male sex, low birth weight, autumn birth, maternal intention to bottle feed, and duration of second stage labour ${ }^{14}$; singletons with a composite score over a cut off point were eligible for inclusion. All multiple births were eligible irrespective of their scores.

Of 13592 liveborn infants in Tasmania in 1988 and 1989, 2791 were eligible for the Tasmanian infant health survey. Of the 1498 infants born in southern Tasmania, a defined geographical region, 1440 (96.1\%) were the subject of a hospital interview soon after the birth. We identified 1185 of these children from school enrolment lists in southern Tasmania, and they were invited to join a study either of childhood blood pressure in 1996 (1988 birth cohort) or of high density lipoprotein cholesterol concentration and blood pressure in 1997 (1989 cohort). All studies were approved by the University of Tasmania ethics committee, and written parental consent was obtained for each subject.

\section{Measurements}

Information on the infants had been collected from hospital obstetric records (gestational age, placental weight, birth weight, crown to heel length, head circumference) and from questionnaires and additional infant anthropometry (skinfold thicknesses) in the postnatal ward, as described previously. ${ }^{13}$

The children were seen at school when they were 8 years of age. Body weight was measured with bathroom scales, which were calibrated daily, and height was measured with a stadiometer. Skinfold thickness was measured with calipers at four sites (triceps, biceps, subscapular, suprailiac), ${ }^{15}$ and right mid upper arm circumference was measured with a tape. Blood pressure was measured three times with an adult and paediatric vital signs monitor (Dinamap, Critikon, Arlington, TX) with the cuff size appropriate for mid upper arm circumference, and the child seated with the left arm resting on a pillow and the elbow approximately at heart level.

Zygosity was assessed from parental reports at 8 years. We asked first whether any tests had been performed and, if so, about the results. If not, we accepted the maternal report of whether the multiplets were identical.

\section{Statistical methods}

We summarised the strength of associations between variables with product moment correlation coefficients and with linear regression coefficients for which the average of three systolic blood pressure values was the dependent variable. We adjusted for putative confounders-including gestational age, mode of delivery, previous pregnancies, duration of breast feeding, maternal smoking and alcohol intake (prenatal and postnatal), maternal education, and child size at age 8-if their association with blood pressure was biologically plausible, and if adjustment substantially changed (by $10 \%$ or more $^{16}$ ) the regression coefficient. To capture the non-linear association of systolic pressure with the measure of fatness in childhood (fat mass estimated from skin folds and body weight, or body mass index), a predictor term for the measure of fatness and another for its square were entered in the same regression model.
For singletons, these statistics were routinely adjusted for four of the six selection factors (maternal age, month of birth, intention to breast feed, and duration of second stage labour) and by stratifying for another (sex). The final selection factor was the study factor, birth weight. Linear predictors were created for month of birth, intention to bottle feed, and duration of second stage labour by scoring their categories with the selection weights for each. Similar adjustments were not routinely made for multiplets because they were not selectively recruited, and there were limited degrees of freedom for those analyses. We checked, however, that artefactual differences had not arisen as a result of unlike methods of analysis. We adjusted for the child's age at blood pressure measurement (as days elapsed since seventh birthday).

In paired analyses, birth weight and blood pressure values from the second born twin were subtracted from corresponding values for the first born twin, and the differences calculated in this way became the subject of statistical inference. Each set of triplets was analysed as three sets of pairs, and within pair differences were calculated as above. To examine the within pair association between birth weight and blood pressure, we regressed the intrapair difference in blood pressure on the intrapair difference in birth weight.

\section{Results}

Subjects

Of the 1185 children from the 1988 ( 572 children) and 1989 (613) Tasmanian infant health survey cohorts identified on school enrolment lists in 1996 and 1997 respectively, we were able to contact 1156 and to obtain measurements from 888 of the original 1185 (74.9\%) children.

Table 1 shows the characteristics of the sample at birth and at a mean age 8.13 years (range 7.26-8.88 years), with subjects classified by sex and whether they were singletons or multiplets. Singletons comprised more boys ( 548 children) than girls (231) because of the weighting given to male sex in the selection criteria of the Tasmanian infant health survey. Comparatively more of the girls $(143 ; 61.9 \%)$ than boys $(296 ; 54.0 \%)$ had birth weights below the 50th centile for gestational age (from norms for singleton births in New South Wales ${ }^{17}$ ). This was because low birth weight was also a selection factor, and its comparative contribution was greater for the girls. The full range of birth weights was nevertheless represented, and $15(6.5 \%)$ girls and $44(8.0 \%)$ boys had birth weights exceeding the 90th centile.

From the 1988 and 1989 cohorts, 128 multiplets were identified at schools in southern Tasmania, and 109 (85.0\%; 70 girls and 39 boys), including two sets of triplets, participated in this study. In five cases the cotwin was not included in the sample (three were sole survivors, one twin was not allowed to participate, and one twin was excluded on medical grounds). To maintain comparability between analyses, these five subjects were excluded. Multiplets had a lower mean birth weight than singletons $(\mathrm{P}<0.01)$ and a shorter mean gestation $(\mathrm{P}<0.01)$, but birth weight adjusted for gestational age was not statistically different: girls $(-108 \mathrm{~g} ; \mathrm{P}=0.11)$, boys $(-132 \mathrm{~g} ; \mathrm{P}=0.15)$. Multiplets 
Table 1 Characteristics of study children

\begin{tabular}{|c|c|c|c|c|c|c|c|c|}
\hline \multirow[b]{3}{*}{ Characteristic } & \multicolumn{4}{|c|}{ Singletons } & \multicolumn{4}{|c|}{ Multiplets } \\
\hline & \multicolumn{2}{|r|}{ Girls } & \multicolumn{2}{|r|}{ Boys } & \multicolumn{2}{|r|}{ Girls } & \multicolumn{2}{|r|}{ Boys } \\
\hline & No & Mean (SD) & No & Mean (SD) & No & Mean (SD) & No & Mean (SD) \\
\hline \multicolumn{9}{|l|}{ At birth } \\
\hline Birth weight $(\mathrm{g})$ & 231 & $2886.4(743.4)$ & 548 & 3282.1 (726.9) & 68 & $2393.9(585.3)$ & 36 & $2370.8(519.3)$ \\
\hline Gestation (weeks) & 231 & $38.2(2.9)$ & 548 & $38.9(2.4)$ & 68 & $36.1(2.7)$ & 36 & $35.1(2.7)$ \\
\hline Length (cm) & 224 & $47.2(3.4)$ & 530 & $49.3(3.1)$ & 64 & $45.1(3.1)$ & 34 & $45.2(2.9)$ \\
\hline Head circumference $(\mathrm{cm})$ & 226 & $33.0(2.2)$ & 529 & $34.3(2.2)$ & 65 & $32.0(2.2)$ & 34 & $31.9(1.9)$ \\
\hline Mid upper arm circumference (mm) & 195 & $10.5(1.3)$ & 480 & $10.9(1.3)$ & 61 & $9.4(1.0)$ & 28 & $9.6(0.8)$ \\
\hline Placental weight $(\mathrm{g})^{*}$ & 229 & $557.8(150.2)$ & 540 & $625.8(158.1)$ & - & - & - & - \\
\hline \multicolumn{9}{|l|}{ At age 8 years } \\
\hline Height $(\mathrm{cm})$ & 231 & $126.8(6.0)$ & 548 & $128.4(6.0)$ & 68 & $128.4(5.7)$ & 36 & $127.0(5.1)$ \\
\hline Weight (kg) & 231 & $27.8(5.7)$ & 548 & $28.1(5.3)$ & 68 & $27.6(4.8)$ & 36 & $26.4(5.4)$ \\
\hline Fat mass $(\mathrm{kg}) \dagger$ & 230 & $6.1(3.6)$ & 548 & $5.5(2.8)$ & 68 & $5.1(2.8)$ & 36 & $5.0(2.9)$ \\
\hline Lean body mass $(\mathrm{kg}) \ddagger$ & 230 & $21.8(2.6)$ & 548 & $22.6(3.0)$ & 68 & $22.4(2.6)$ & 36 & $21.4(2.9)$ \\
\hline Systolic blood pressure (mm Hg) & 231 & $108.3(9.1)$ & 548 & $107.3(8.9)$ & 68 & $110.4(9.2)$ & 36 & $109.3(11.2)$ \\
\hline Diastolic blood pressure $(\mathrm{mm} \mathrm{Hg})$ & 231 & $64.3(6.6)$ & 548 & $63.6(6.4)$ & 68 & $65.0(7.1)$ & 36 & $64.2(7.3)$ \\
\hline
\end{tabular}

*Placental weight only reported for singletons because individual placental weights are unreliable in multiplets.

tCalculated from sum of four skinfold thicknesses.

$\ddagger$ Calculated as weight $(\mathrm{kg})$ minus fat mass $(\mathrm{kg})$.

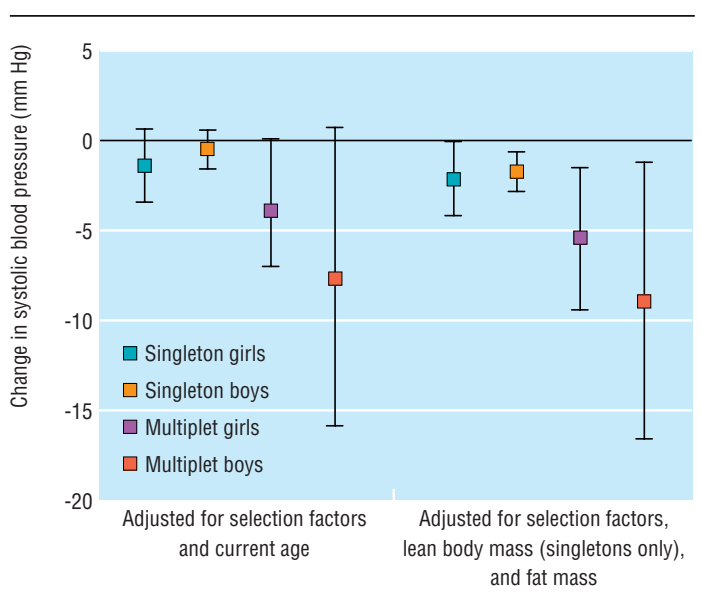

Effect of $1 \mathrm{~kg}$ increase in birth weight on systolic blood pressure at age 8 years, with and without adjustment for current size

had higher mean systolic blood pressure by around $2 \mathrm{~mm} \mathrm{Hg}(\mathrm{P}<0.01)$ than singletons.

\section{Birth weight and childhood blood pressure}

Systolic pressure was negatively associated with birth weight. Children with greater lean or fat mass at age 8 years had higher blood pressure and had been heavier at birth than smaller children at age 8 years. Adjusting for childhood fat and lean mass (the childhood size measures most strongly associated with blood pressure) removed the confounding effect of current size, thereby increasing the estimated strength of the association between systolic pressure and birth weight (figure). We adjusted only for fat mass in multiplets because of the colinearity of the fat mass and lean mass measurements for these children.

The association was stronger in multiplets than in singletons. For each $1 \mathrm{~kg}$ increase in birth weight there was an estimated change in systolic pressure of -1.94 $\mathrm{mm} \mathrm{Hg}(95 \%$ confidence interval -2.89 to -0.98$)$ in singletons (girls: $-2.16,-4.24$ to -0.08 ); boys: -1.73 , -2.83 to -0.64$)$ and $-7.0 \mathrm{~mm} \mathrm{Hg}(-10.1$ to -3.9$)$ in multiplets (girls: $-5.9,-9.6$ to -2.2 ; boys: -8.4 , -15.6 to -1.2 ). Estimates for singletons, but not multi- plets, were routinely adjusted for the selection criteria factors. Similar adjustment for multiplets made little difference: adjusted estimates were $-5.4 \mathrm{~mm} \mathrm{Hg}(-9.4$ to -1.5$)$ for girls and $-8.9(-16.5$ to -1.2$)$ for boys. The estimated fall in systolic pressure per $1 \mathrm{~kg}$ increase in birth weight was greater for multiplets than for singletons (for both sexes $\mathrm{P}<0.01$, girls $\mathrm{P}=0.14$, boys $\mathrm{P}=0.02$ ). We found no significant association between birth weight and diastolic pressure.

We searched for factors that might explain the different effect size in multiplets and singletons. Gestational age did not influence systolic pressure independently of birth weight, and adjustment for it left almost unaltered the estimated effect of birth weight in multiplets. They were more commonly delivered by caesarean (26 of 104 (25\%) versus 127 of 778 $(16.3 \%)$ singletons), and delivery of a further 15 multiplets was medically or surgically induced before full term. Removing these two groups made little difference: the birth weight effect among the 65 remaining multiple births was $-8.9 \mathrm{~mm} \mathrm{Hg}(-13.1$ to -4.6) per $1 \mathrm{~kg}$. Adjusting for number of previous pregnancies, breast feeding, maternal smoking and alcohol intake (prenatal and postnatal), or maternal education did not substantially change (by as much as $10 \%)$ the difference in the estimated effect of birth weight on blood pressure between multiplets and singletons.

\section{Within pair analyses in multiplets}

In multiplets the size of the birth weight effect was largely unchanged in within pair analyses in which we regressed the within pair difference in blood pressure on the within pair difference in birth weight (table 2). Estimates were generally larger for monozygotic than for dizygotic pairs, but differences were not statistically significant in this sample size. To provide greater depth to the analysis, we investigated different birth measures. The same pattern of effects and persistence of effect size after pairing was seen for crown to heel length. Associations were weaker for arm and head circumferences, body size ratios, and infant skin fold thicknesses (data not shown). 
Table 2 Influence of birth weight and birth length on systolic blood pressure in multiplets, at 8 years of age

\begin{tabular}{|c|c|c|c|}
\hline Change in systolic pressure $(\mathrm{mm} \mathrm{Hg})$ & All multiplets & Monozygotes & Dizygotes \\
\hline \multicolumn{4}{|l|}{ Before pairing* } \\
\hline & $\mathrm{n}=104$ & $\mathrm{n}=32$ & $\mathrm{n}=72$ \\
\hline \multirow[t]{2}{*}{$1 \mathrm{~kg}$ increase in birth weight } & $-7.0(-10.1$ to -3.9$)$ & $-10.4(-19.1$ to -1.7$)$ & $-6.4(-9.7$ to -3.1$)$ \\
\hline & $\mathrm{n}=98$ & $n=29$ & $n=69$ \\
\hline $10 \mathrm{~cm}$ increase in birth length & $-10.7(-17.1$ to -4.3$)$ & $-17.6(-36.2$ to 0.9$)$ & $-9.3(-16.1$ to -2.6$)$ \\
\hline \multicolumn{4}{|l|}{ Within pair analyses $\dagger$} \\
\hline & $n=55$ & $n=16$ & $\mathrm{n}=39$ \\
\hline \multirow[t]{2}{*}{$1 \mathrm{~kg}$ increase in birth weight } & $-5.3(-13.8$ to 3.2$)$ & $-6.5(-22.5$ to 9.4$)$ & $-4.9(-15.8$ to 6.0$)$ \\
\hline & $\mathrm{n}=51$ & $\mathrm{n}=14$ & $\mathrm{n}=37$ \\
\hline $10 \mathrm{~cm}$ increase in birth length & $-5.6(-19.2$ to 8.0$)$ & $-30.6(-66.9$ to 5.7$)$ & $-2.3(-17.8$ to 13.1$)$ \\
\hline
\end{tabular}

${ }^{*}$ Adjusted for current age and fat mass (calculated from sum of four skinfold thicknesses).

†Adjusted for within pair difference in fat mass.

\section{Discussion}

This is the first report of an association between birth weight and blood pressure in children from multiple pregnancies. We found a stronger negative association among multiplets than singletons. This observation in itself has the potential to provide clues to the origin of the relation between birth weight and blood pressure. More importantly, the strong association among multiplets afforded an opportunity to gain insights, through within pair analyses, into the role that exposures originating with the mother played, and also the likely contribution of genetic factors.

Implications of within pair analyses in multiplets The association between birth weight and blood pressure, seen in multiplets as a group, was not diminished in within pair analyses. This implies that maternal factors, including nutrient intake, cannot be directly responsible for the association between birth weight and blood pressure. The critical step in the causal pathway must concern mechanisms that lie within the fetoplacental unit, with maternal exposures operating only as antecedents or primers in the causal pathway. This is an important conclusion, which is contrary to the current paradigm in this research field. This key observation is supported by the work of Poulsen et al, ${ }^{18}$ who found an association between birth weight and type 2 diabetes in twins, which remained after within pair analysis.

Although the sample size (over 100 multiplets) provided sufficient power to determine whether the effect remained after pairing, it was insufficient to permit estimation of the difference in effect between monozygotic and dizygotic twins either before or after pairing. None the less, the strong association was comparatively unchanged in within pairs analysis in monozygotic twins, implying that the association between birth weight and blood pressure does not originate in the genes of the infant.

A possible explanation for the association is that birth size itself is a component of the principal causal pathway that leads to later high blood pressure and possibly other adverse metabolic outcomes. That the strength of the association between birth weight and blood pressure was greater in multiplets than singletons would, however, make that unlikely, unless some confounding or effect modifying factor was operating differentially in multiplets and singletons.

\section{The different association in multiplets and} singletons

The finding of a stronger association between birth weight and childhood blood pressure in multiplets than in singletons may provide a clue as to what may be responsible for the association between birth weight and blood pressure, but can we be confident of the observation? It cannot be accounted for by an unusually weak association in this study among singletons. The estimated effect of birth weight on systolic pressure in the 8 year old singletons, at around -2 $\mathrm{mm} \mathrm{Hg}$ per $1 \mathrm{~kg}$ increase in birth weight, was within the range of effect sizes estimated in other studies of children of comparable age in Australia, ${ }^{19}$ the United Kingdom, ${ }^{20-22}$ and Zimbabwe. ${ }^{23}$ We found slightly larger effects for singleton girls $(-2.16 \mathrm{~mm} \mathrm{Hg}$ per 1 $\mathrm{kg}$ ) than singleton boys $(-1.73 \mathrm{~mm} \mathrm{Hg}$ per $1 \mathrm{~kg})$, in common with other studies. ${ }^{20}{ }^{21}{ }^{24}$ It is also unlikely to be explained by the sample selection process. Although singletons in the Tasmanian infant health survey were not representative of the total infant population, analyses were adjusted for factors used as selection criteria, and the estimated effect size in singletons was very similar to that in other populations. Multiplets were not subject to any selection bias as all born in the study period were eligible and the response at follow up was high (85.0\%), as in singletons $(74.9 \%)$.

The stronger association in multiplets raises the hypothesis that of the potential causes of low birth weight or later high blood pressure those that influence both were more prevalent in multiplets. What factors then within the fetoplacental unit could be associated with birth weight and blood pressure and also more prevalent in multiple pregnancies? Supply of oxygen and nutrients across the placenta can be unequally distributed between individual multiplets, and reduced oxygen or nutrient availability may be more prevalent causes of growth restriction in multiplets than in singletons and lead to permanent adverse programming of the cardiovascular system. We were unable to address these questions in any depth, but we did examine the association between birth weight and blood pressure in singletons before and after controlling for placental weight. The addition of placental weight to the regression model had little effect on the estimates, but the weight of the placenta may not reflect its function. 
Key messages

- Low birthweight is associated with high blood pressure from an early age

- Maternal factors, including diet during pregnancy, cannot wholly explain the association between fetal development and later risk of cardiovascular disease

- Because the association between blood pressure and birthweight remained in monozygotic twins, genetic factors are unlikely to be responsible for the association

- Important causes of the association seem to be operating within the fetoplacental unit during fetal life

- Further research should now focus on placental function and fetal exposures

\section{Conclusion}

These findings need to be confirmed in other studies and for other risk factors for adult cardiovascular disease. If replicated they would support a shift in the thrust of future research from a focus on maternal nutrition as a cause of the association between birth weight and blood pressure and possible cardiovascular disease to factors within the fetoplacental unit.

We thank the nurses who measured blood pressures; the children and their families; and the schools.

Contributors: TD conceived the idea for this study, and both he and A-LP contributed to its design. All authors participated in the analysis or interpretation of data, or both, and in drafting or revising the manuscript, or both, and each approved the version published. TD and LB will act as guarantors for the paper.

Funding: This study was funded by the National Health and Medical Research Council of Australia. The Tasmanian infant health survey was funded by the National Health and Medical Research Council of Australia, US National Institutes of Health (grant 001 HD28979-01A1, Tasmanian State Government, Australian Rotary Health Research Fund, Sudden Infant Death Syndrome Research Foundation, National Sudden Infant Death Syndrome Council of Australia, Community Organisations' support programme of the Department of Human Services and Health, Zonta International, Wyeth Pharmaceuticals, and Tasmanian Sanatoria After-Care Association. A-LP was supported by a National Health and Medical Research Council Public Health Research and Development Committee fellowship.

Competing interests: None declared.
1 Law CM, de Swiet M, Osmond C, Fayers PM, Cruddas AM, Fall CH. Initiation of hypertension in utero and amplification throughout life. $B M$ 1993;306:24-7.

2 Leon DA, Lithell HO, Vagero D, Koupilova I, Mohsen R, Berglund L, et al Reduced fetal growth rate and increased risk of death from ischaemic heart disease: cohort study of 15000 Swedish men and women born 1915-29. BMJ 1998:317:241-5

3 Hales CN. Non-insulin-dependent diabetes mellitus. Brit Med Bull 1997;53:109-22.

4 Hofman PL, Cutfield WS, Robinson EM, Bergman RN, Menon RK, Sperling MA, et al. Insulin resistance in short children with intrauterine growth retardation. J Clin Endocrinol Metab 1997;82:402-6.

5 Yajnik CS, Fall CH, Vaidya U, Pandit AN, Bavdekar A, Bhat DS, et al. Fetal growth and glucose and insulin metabolism in four-year-old Indian children. Diabet Med 1995;12:330-6.

6 Barker DJP. The fetal and infant origins of adult disease. BMJ 1990;301:1111.

7 Barker DJP, Gluckman PD, Godfrey KM, Harding JE, Owens JA, Robinson JS. Fetal nutrition and cardiovascular disease in adult life. [Review.] Lancet 1993;341:938-41.

8 Phillips DI. Insulin resistance as a programmed response to fetal undernutrition. Diabetologia 1996;39:1119-22.

9 Lucas A. Programming by early nutrition: an experimental approach J Nutr 1998;128:401-6S.

10 Langley-Evans SC, Gardner DS, Welham SJ. Intrauterine programming of cardiovascular disease by maternal nutritional status. Nutrition 1998:14:39-47.

11 Kramer MS. Determinants of low birth weight: methodological assessment and meta-analysis. Bull WHO 1987;65:663-737.

12 Gliksman MD, Kawachi I, Hunter D, Colditz GA, Manson JE, Stampfer MJ, et al. Childhood socioeconomic status and risk of cardiovascular disease in middle aged US women: a prospective study. J Epidemiol Community Health 1995;49:10-5

13 Dwyer T, Ponsonby A-L, Newman NW, Gibbons LE. Prospective cohort study of prone sleeping position and sudden infant death syndrome. Lancet 1991;337:1244-7.

14 d'Espaignet ET, Dwyer T, Newman NM, Ponsonby AL, Candy S. The development of a model for predicting infants at high risk of sudden infant death syndrome in Tasmania. Paediatr Perinat Epidemiol $1990 \cdot 4 \cdot 422-35$

15 Dwyer T, Blizzard CL. Defining obesity in children by biological endpoint rather than population distribution. Int J Obes 1996;20:472-80.

16 Greenland S. Modeling and variable selection in epidemiologic analyses. Am J Public Health 1989;79:340-9.

17 Beeby PJ, Bhutap T, Taylor LK. New South Wales population-based birth weight percentile charts. J Paediatr Child Health 1996;32:512-8.

18 Poulsen P, Vaag AA, Kyvik KO, Moller Jensen D, Beck-Nielsen H. Low birth weight is associated with non-insulin dependent diabetes mellitus in discordant monozygotic and dizygotic twin pairs. Diabetologia 1997;40:439-46.

19 Moore VM, Miller AG, Boulton TJ, Cockington RA, Craig IH, Magarey $\mathrm{AM}$, et al. Placental weight, birth measurements, and blood pressure at age 8 years. Arch Dis Child 1996:74:538-41.

20 Whincup PH, Cook DG, Shaper AG. Early influences on blood pressure: a study of children aged 5-7 years. BMJ 1989;299:587-91.

21 Taylor SJ, Whincup PH, Cook DG, Papacosta O, Walker M. Size at birth and blood pressure: cross sectional study in 8-11 year old children. $B M$ J 1997;314:475-80

22 Whincup P, Cook D, Papacosta O, Walker M. Birth weight and blood pressure: cross sectional and longitudinal relations in childhood. $B M$ 1995;311:773-6.

23 Woelk G, Emanuel I, Weiss NS, Psaty BM. Birth weight and blood pressure among children in Harare, Zimbabwe. Arch Dis Child Fetal Neonatal Edition 1998;79:119-22F.

24 Barker DJ, Osmond C, Golding J, Kuh D, Wadsworth M. Growth in utero, blood pressure in childhood and adult life, and mortality from cardiovascular disease. BMJ 1989;298:564-7.

(Accepted 19July 1999)

\section{Email submissions from outside the United Kingdom}

We are now offering an email submission service for authors from outside the UK. The address is papers@bmj.com

Ideally our email server would link seamlessly with our manuscript tracking system, but for now it does not, which is why we are offering the service only to authors outside the UK. Most post in the UK arrives the next day, so UK authors have the least to gain in speed of delivery from email delivery. As soon as our systems improve we will invite email submissions from everyone.

If you choose to send your submission by email please would you send the text and any tables and figures as attached files, together with a covering letter giving all your contact details (postal address, phone, fax, and email address). We can read files created with most word processing, graphics, and spreadsheet programs.

When your submission is received in our email box you will receive an automatic acknowledgment to show that it has arrived. If the submission is incomplete we will contact you and ask you to resend the missing information.

Once the submission is complete we will register it on our manuscript tracking system and you will receive a standard acknowledgment in the post.

Letters to the editor should continue to be sent direct to www.bmj.com as rapid responses or to letters@bmj.com 